\title{
Modeling and Validation of the Layered Deformation for Double Sided Incremental Forming
}

\author{
Yu-Huai Wang ${ }^{1,2, *}$, Wei Peng ${ }^{1}$, Qiu-Cheng Wang ${ }^{1}$ and Jiang Cao ${ }^{1}$ \\ ${ }^{I}$ Key Laboratory of E\&M (Zhejiang University of Technology), Ministry of Education \& Zhejiang Province, Hangzhou, \\ P.R. China \\ ${ }^{2}$ Qianjiang College, Hangzhou Normal University, Hangzhou, P.R. China
}

\begin{abstract}
This work proposes an equivalent layered model based on the contour trajectory for Double Sided Incremental Forming (DSIF). The layered deformation and displacement are analyzed for the truncated cones with different height. The displacement for every layer is obtained by numerical analysis and theoretical calculation under the assumption of straight projection. A general method is introduced to estimate the thickness by using of the surface geometry of the desired component. Analytical, numerical and experimental results are compared for the historical displacement, the final geometry and thickness. It is demonstrated that the proposed model and method are reliable and effective in the predictions of historical displacement, geometric accuracy and thickness for DSIF.
\end{abstract}

Keywords: Incremental forming, equivalent layered model, displacement, thickness prediction.

\section{INTRODUCTION}

Incremental forming (IF) is a flexible sheet forming process for rapid prototyping and small batch and customized production without traditional dies where the blank clamped in a blankholder is progressively deformed into the desired shape by one or more generic tools traveling along a predefined path. Single Point Incremental Forming (SPIF) that employs a single tool to form the sheet from one side is the most common configuration of IF. SPIF is plagued by an inherent geometric inaccuracy [1]. Allwood et al. [2] report that the accuracy of IF processes is only $\pm 3 \mathrm{~mm}$, and the required accuracy is around $\pm 0.2 \mathrm{~mm}$ for industrial application. $\mathrm{Lu}$ et al. [3] developed a feature-based tool path generation method according to the specified critical edges to obtain better geometric accuracy. Rauch $\mathrm{M}$ et al. [4] tested three tool path types and proposed intelligent CAM approach to generate tool path. However, they find that the free area of the sheet has plastic deformation and the final height is lower than the desired value, because of the springback and the shape of the sheet clamped system. Tanaka et al. [5] used a numerical model to study the residual stresses and came to the conclusion that the springback is caused by the residual moments. Allwood et al. [6] pointed out that the sheet deformation is not determined entirely by the tool path. So, they cut slots and tabs to form a partially cut-out blank by a laser cutter to improve the geometric accuracy. The more accurate parts can be obtained by the use of partially cut-out blanks than by the use of SPIF without backing plate,

*Address correspondence to this author at the Key Laboratory of E\&M (Zhejiang University of Technology), Ministry of Education \& Zhejiang Province, Hangzhou, P.R. China; Tel: 13588882341;

E-mail:wyiya@hotmail.com because of the localized deformation shown in (Fig. 1). Conversely, SPIF with backup plate which prevents the flexibility give more accurate parts than partially cut-outs.

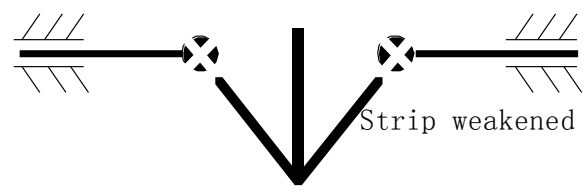

Fig. (1). the localize deformation under point load for partially cutout blank.

To preserve the process flexibility and to improve geometric accuracy, double-sided IF (DSIF) is proposed and developed by introduction of the concept intending to localize deformation. Tisza et al. [7] employed a slave tool synchronized with the master tool to provide a flexible support. They find that the slave tool will lose the contact with sheet after a period of time during the forming process. Meier et al. [8] used two robots to mount an upper and lower tool. Franzen et al. [9] used an exchangeable support tool fixed on a rotating plate as dynamic support. They have shown that the minimum and maximum deviation is $-0.27 \mathrm{~mm}$ and $0.91 \mathrm{~mm}$, respectively. Malhotra et al. [1] studied in-to-out tool path strategy to maintain the contact between forming tool and supporting tool which are displacement controlled and improve the accuracy. WANG et al. [10] used two pairs of tools as the dynamic supporting tools which are located on the back and side of a pair of main tools to localize the deformation and developed experimental equipment. Many researches $[11,12]$ predicted and discussed the final shapes 
from the undeformed sheet by experimental measurements and FEM simulations for SPIF and Kinematics ISF. Bambach M [12] introduced idealized intermediate shapes of which the deformation proceeds by displacements along the surface normal to compute the trajectories of surface points. It yields better results than the sine law. However, it is necessary to take into account the mechanical equilibrium and the material behavior.

In this paper, a layered deformation model and algorithm is presented based on an equivalent layered model. Firstly, an equivalent model with contour layer is introduced based on the tool contour trajectory and a general thickness calculation method is proposed by the surface of arbitrary geometries. Secondly, the process of deformation is analyzed under point load and tools for the truncated cones. The relationship of displacement and contour forming layer is calculated by using of the translation of arc determined by the radius of tool. The thickness calculation method is consistent with sine law for cone. Finally, Numerical and experimental studies are conducted to validate the presented model and method. The analytical and numerical results of the entire historical displacement, geometric accuracy and thickness are reasonably good agreement with experimental results for the conical shapes with $1 \mathrm{~mm}$ Al5052.

\section{DEFORMATION PREDICTION ALGORITHM}

\subsection{The Contour Trajectory and Thickness Analysis}

Let's suppose that the surface geometry of the desired component is represented by function $F(x, y, z)=0$. When $z=h$, the tool contour trajectory can be expressed as

$F(x, y, h)=0$

The model of contour trajectory in incremental forming is illustrated in (Fig. 2).

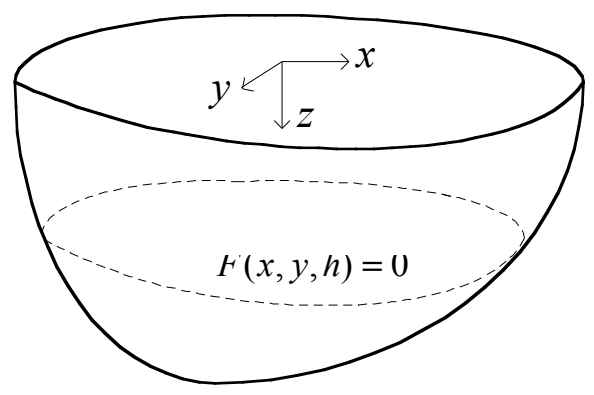

Fig. (2). Model of contour trajectory.

Suppose that the deformation proceeds along the normal of the tool contour plane. Due to volume constancy, we obtain

$t_{0} d x d y=t d A$

Where, $t_{0}$ and $t$ are the initial thickness and actual thickness of sheet, respectively. $d x$ and $d y$ are the length of microbody along $x$-axis and $y$-axis, respectively. $d A$ is the deformed area element.
According to the formula for the surface area,

$d A=\sqrt{1+\left(\frac{\partial z}{\partial x}\right)^{2}+\left(\frac{\partial z}{\partial y}\right)^{2}} d x d y$

Substitution of Eq. (3) into Eq. (2) gives

$t=t_{0} / \sqrt{1+\left(\frac{\partial z}{\partial x}\right)^{2}+\left(\frac{\partial z}{\partial y}\right)^{2}}$

\subsection{The Equivalent Layered Model Based on Contour Trajectory}

It is difficult to calculate the real state of deformation at a certain instant, because of the very complex threedimensional forming process for DSIF. To obtain the rule of the deformation to guide the planning of tool path and the design of process parameters during forming, a concept of the equivalent model with contour layer is introduced to simplify the complex forming process.

Definition: The equivalent layered model is a virtual load model that corresponds to the cumulative plastic strain increment $\varepsilon_{p}$ for a whole formed contour layer.

For simplicity, the equivalent model with contour layer for forming of a truncated cone with the top tool as the forming tool and the bottom tool which is far away from top tool with distance $d$ as the dynamical support tool is shown in (Fig. 3). The equivalent forming and constraint forces of the model are virtual and axisymmetric for a whole contour layer. The plastic strain is equal to the above mentioned cumulative plastic strain increment $\varepsilon_{p}$ within the neighborhood of a geometry layer. The total plastic strain is equivalent. Therefore, the equivalent layered model which is a virtual load model can be used to analyze the deformation process in detail.

If the axial increment is $d z$, according to Eq. (1), the tool contour trajectory for the $n$th layer is $F(x, y, n d z)=0$.

In reference to (Fig. 3), the $n$th contour for a cone can be expressed as

$L_{n}:\left\{\begin{array}{l}x=r_{n} \cos \varphi \\ y=r_{n} \sin \varphi \\ z=n \cdot d z\end{array}\right.$

Where, $r_{n}$ is the distance from the central axis to the axis of forming head for the $n$th layer.

\subsection{The Layered Deformation History Analysis and Cal- culation for Cone}

Let the height and wall angle of the forming cone are $H$ and $\alpha$ respectively. Then, the total forming layer $N$ is

$N=H / d z$

So, the radial increment $d r$ is

$d r=\frac{r_{0}-r_{N}}{N}=\frac{\left(r_{0}-r_{N}\right) d z}{H}=d z \tan \alpha$

$r_{n}=r_{0}-n d r$ 


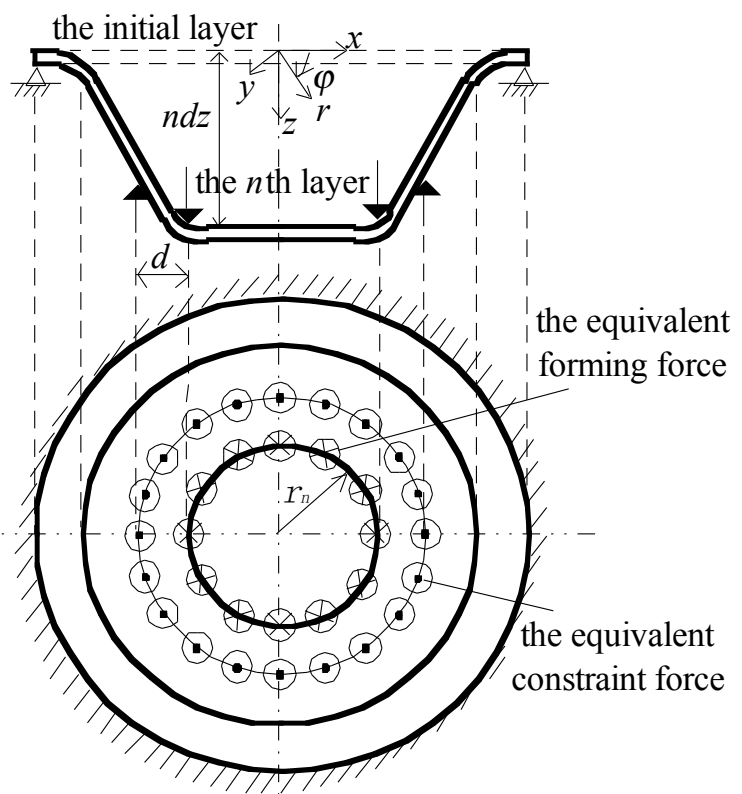

Fig. (3). The equivalent model with contour layer for a truncated cone.

Under point load, the layered deformation process is shown as (Fig. 4) for DSIF.

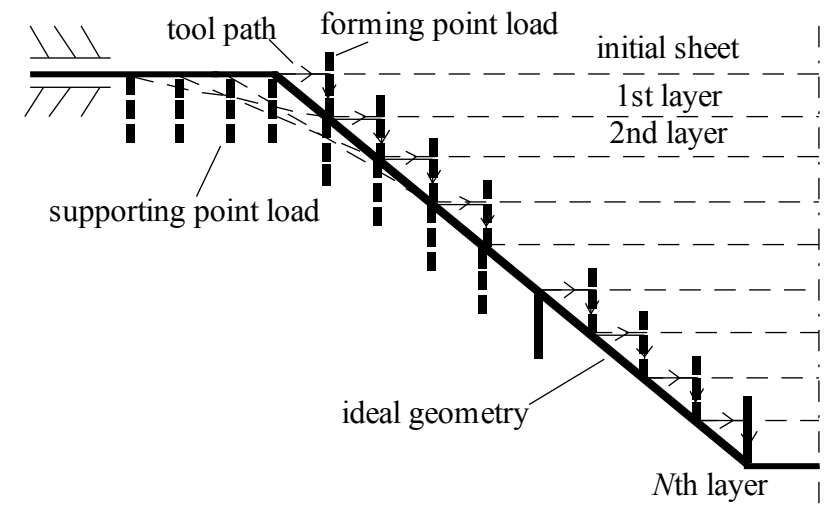

Fig. (4). The process of layered deformation under point load.

Considering the radius of tools $r_{T}$ with hemispherical head, the layered deformation process is as follows. The bending arc is uniquely determined by the radius of tools (Fig. 5).

Definition: While forming in arbitrary layer, the points, located in the outer side of main forming tool, in the formed area, are called external points. On the other hand, the points located in the inner side and unformed area, are called interior points.

In cylindrical coordinates, an arbitrary point $P(r, \varphi, 0)$ on the initial blank is finally projected to $Q(r, \varphi, z)$ along z-axis based on the straight projection and plane strain deformation.

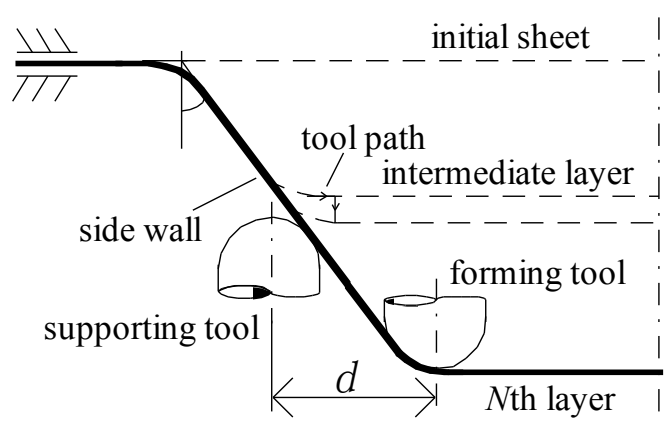

Fig. (5). the process of the layered deformation.

Let $u_{k}(r)$ be the axial displacement of the point $P$ for the $k t h(k \in[0, N])$ layer, $r_{k}$ be the distance from the central axis to the axis of forming head for the $\mathrm{k} t h$ layer.

Let $P$ be the interior point for $\mathrm{n} t h$ layer and the external point for $(\mathrm{n}+1)$ th layer. That is

$r_{n+1} \leq r<r_{n}$

According to Eq.(8), we have

$\frac{r_{1}-r}{d r} \leq n<\frac{r_{0}-r}{d r}$

Hence,

$n=\left\{\frac{r_{1}-r}{d r}\right\}$

Where, \{\} rounds to the nearest integers towards infinity.

So, the $u_{k}(r)$ can be expressed as

$u_{k}(r)=\left\{\begin{array}{ccl}k \cdot d z & k \in[0, n] \\ \sqrt{r_{T}^{2}-\left(r-r^{\prime}\right)^{2}}+k d z-r_{T} & k \in(n, N] & \text { and } r<r^{\prime}+r_{T} \cos \alpha \\ \left(r_{0}-r\right) / \tan \alpha & k \in(n, N] & \text { and } r \geq r^{\prime}+r_{T} \cos \alpha\end{array}\right.$

Eq. (11)

Where, $r^{\prime}=r_{k}-r_{T} \tan \left(45^{\circ}-\alpha / 2\right)$.

The resultant displacement can be expressed as

$\Delta u(r)=u_{k+1}(r)-u_{k}(r)$

Especially, the external points for $1^{\text {st }}$ layer, that is $r_{1}+d \leq r_{x}$, we have

$u_{x}=0$

And the interior point for $N$ th layer, that is $r_{x} \leq r_{N}$, we have

$u_{x}=N \cdot \Delta h$

Eq. (14)

\subsection{Thickness Analysis for Cone}

The surface equation of cone can be expressed as 


$$
z=\left(R-\sqrt{x^{2}+y^{2}}\right) \cot \alpha
$$

The partial derivative can be calculated as

$$
\left\{\begin{array}{l}
\frac{\partial z}{\partial x}=\frac{-x \cot \alpha}{\sqrt{x^{2}+y^{2}}} \\
\frac{\partial z}{\partial y}=\frac{-y \cot \alpha}{\sqrt{x^{2}+y^{2}}}
\end{array}\right.
$$

Plugging Eq. (16) into Eq. (4), the sheet thickness can be calculated as

$$
t=t_{0} / \sqrt{1+\cot ^{2} \alpha}=t_{0} \sin \alpha
$$

Thus, it can be seen that the aforementioned thickness prediction method is agreement with the sine law (Matsubara, 1994) for cone.

\section{COMPARISON OF THEORETICAL PREDICTION WITH NUMERICAL SIMULATIONS AND EXPERI- MENTAL RESULT}

To verify the effectiveness of the proposed model and method, numerical simulations are conducted using Ls-dyna and the results are compared against the analytical and experimental results. Two kinks of cones with wall angles of $76^{\circ}$ and $53.2^{\circ}$ were formed on the sheet of thickness $1.0 \mathrm{~mm}$ by adopting two contour paths with axial increment $d z=0.5 \mathrm{~mm}$, radial increment $d r=2 \mathrm{~mm}$ and $d z=0.75 \mathrm{~mm}$, $d r=1 \mathrm{~mm}$ respectively. The diameter of the blank sheet with density $2750 \mathrm{Kg} / \mathrm{m} 3$, Modulus of elasticity 70GPa, Poisson's ratio 0.33 , yield strength $150 \mathrm{MPa}$ and Tangent modulus $277 \mathrm{MPa}$ is $160 \mathrm{~mm}$. The diameter of tools treated as a rigid body is $6 \mathrm{~mm}$. For the $76^{\circ}$ cone, geometry parameters are: $r_{0}=40 \mathrm{~mm}, r_{N}=20 \mathrm{~mm}, H=5 \mathrm{~mm}$. For the $53.2^{\circ}$ cone, geometry parameters are: $r_{0}=30 \mathrm{~mm}, r_{N}=10 \mathrm{~mm}, H=15 \mathrm{~mm}$. Two kinks of cones simulated in Ls-dyna are shown in (Figs. 6 and 7).

The experiment is performed in a custom-designed machine shown in (Fig. 8). The experimental parameters are same as those used in simulation. Components formed with DSIF are shown in (Fig. 9).

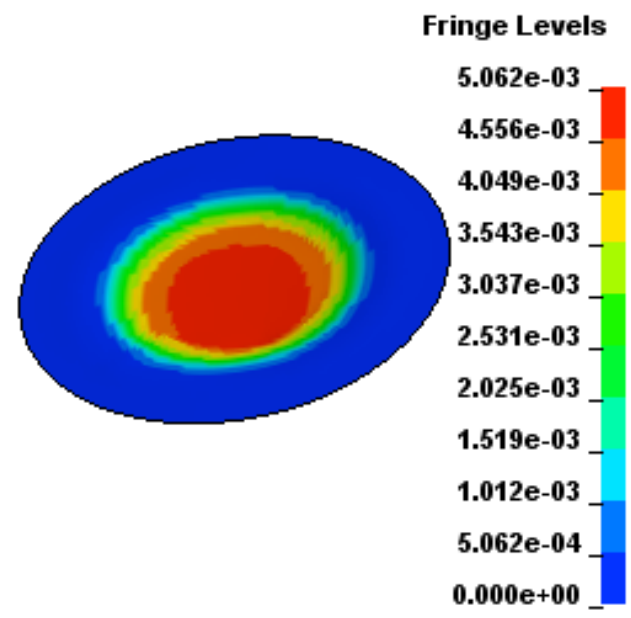

Fig. (6). Simulated components in Ls-dyna for $76^{\circ}$ cone.

\section{Fringe Levels}

$1.492 \mathrm{e}+01$

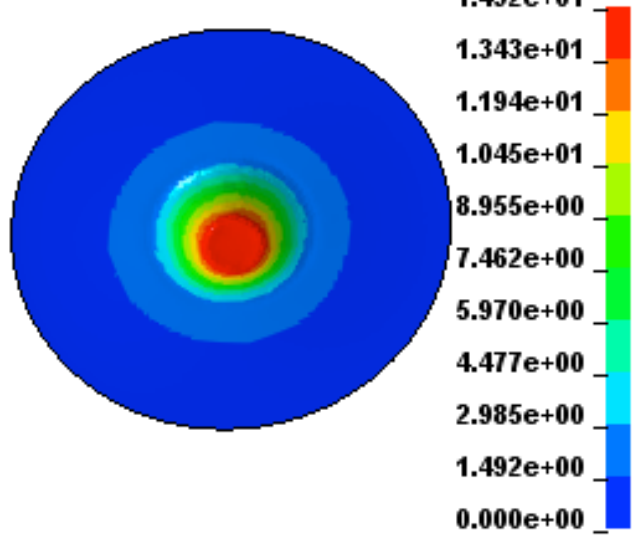

Fig. (7). Simulated components in Ls-dyna for $53.2^{\circ}$ cone.

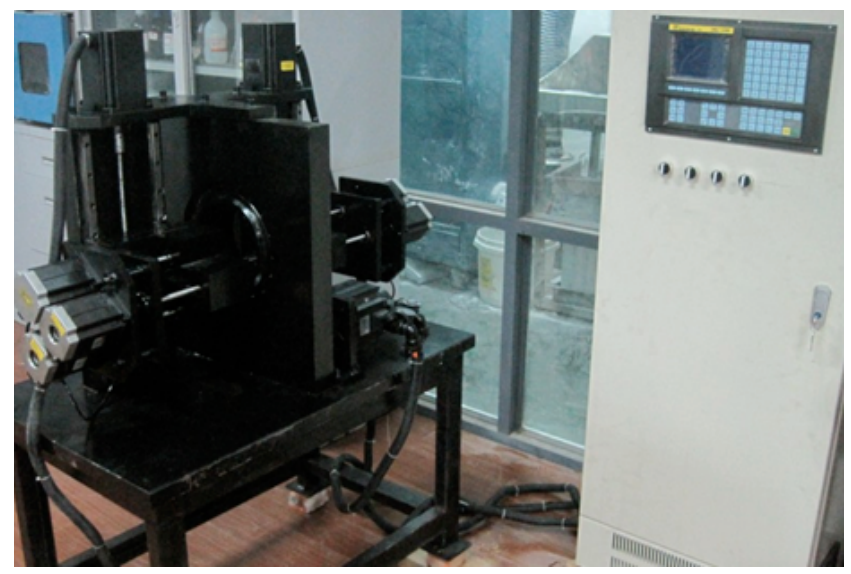

Fig. (8). Fabricated forming machine.

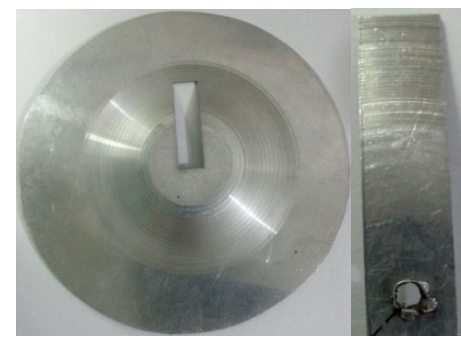

(a)

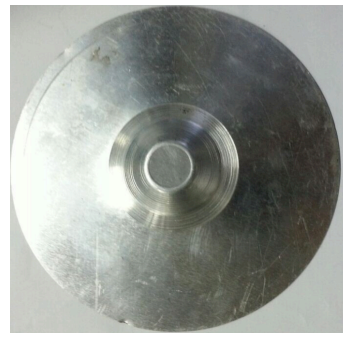

(b)
Fig. (9). Formed components for (a) $76^{\circ}$ cone and wire-Cutting specimen and (b) $53.2^{\circ}$ cone.

\subsection{Historical Displacement}

The numerical historical displacement which is extracted from displacement fields simulated by using Finite Element Method (FEM) was compared to the analytical displacement calculated by Eq. (11).

For $76^{\circ}$ and $53.2^{\circ}$ cone, the axial displacement with $\mathrm{r}=20 \mathrm{~mm}$ and $\mathrm{r}=21 \mathrm{~mm}$ is shown as Fig. (10) and Fig. (11), respectively. 


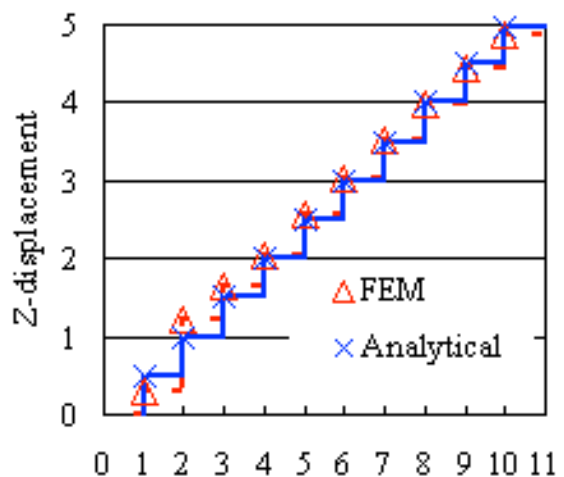

Layer number

Fig. (10). The axial historical displacement for $76^{\circ}$ cone with $\mathrm{r}=20 \mathrm{~mm}$.

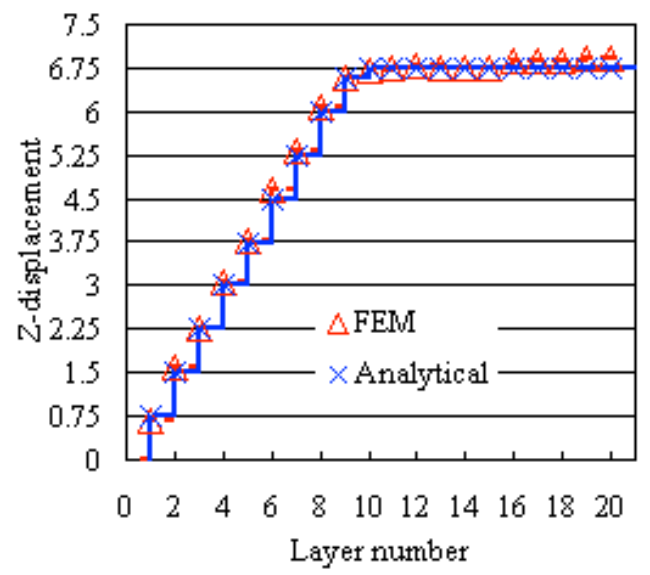

Fig. (11). The axial historical displacement for $53.2^{\circ}$ cone with $\mathrm{r}=21 \mathrm{~mm}$.

It can be seen that, (1) the deformation is incremental and layered; (2) the forming waiting area will produce the predeformation; (3) the formed area is minimally affected by the subsequent processing layer; (4) the axial increment has major effect on the axial historical displacement. The smaller the axial increment is, the more layers are, the smaller the displacement of each layer is; (5) the total height is always lower than the ideal value. This comes from the springback.

\subsection{Geometry Accuracy}

The geometries of the formed cones of which the profile surface was scanned using the Faro Gage after removing the tools and unclamping the sheet were compared to the ideal geometries and simulation geometries in (Fig. 12 and 13).

Fig. (12) shows that the starting deformation occurred at $\mathrm{x}=40 \mathrm{~mm}$ ideally occurred at $51.862 \mathrm{~mm}$ for experiment and at $57.34 \mathrm{~mm}$ for simulation actually for $76^{\circ}$ cone. (Fig. 13) shows that the starting deformation occurred at $\mathrm{x}=30 \mathrm{~mm}$ ideally occurred at52.294mm for experiment and at $52.332 \mathrm{~mm}$ for simulation actually for $53.2^{\circ}$ cone.

The fitted wall angles are listed in Table 1 for the simulation and experimental cones by use of the least square method.

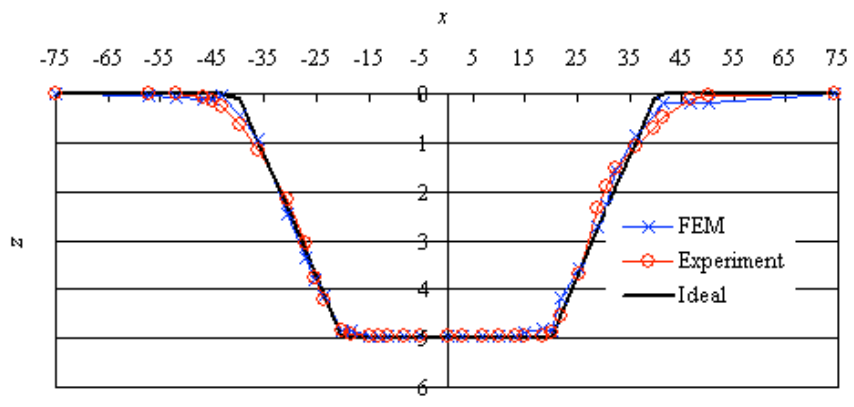

Fig. (12). Comparison of ideal, numerical and experimental geometries for $76^{\circ}$ cone.

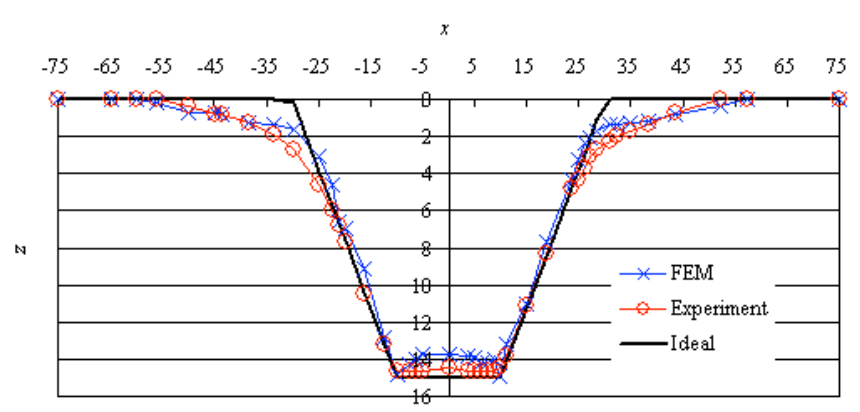

Fig. (13). Comparison of ideal, numerical and experimental geometries for $53.2^{\circ}$ cone.

Table 1. Comparison of ideal, numerical and experimental wall angle.

\begin{tabular}{|c|c|c|}
\hline & $\begin{array}{c}\text { wall angle for } \mathbf{7 6}^{\circ} \\
\text { cone }\end{array}$ & $\begin{array}{c}\text { wall angle for } \mathbf{5 3 . 2 ^ { \circ }} \\
\text { cone }\end{array}$ \\
\hline \hline Ideal & $76^{\circ}$ & $53.2^{\circ}$ \\
\hline FEM $(x<0)$ & 77.008 & 55.264 \\
\hline $\operatorname{FEM}(x>0)$ & 76.996 & 56.006 \\
\hline Experiment $(x<0)$ & $77.369^{\circ}$ & 57.709 \\
\hline Experiment $(x>0)$ & 76.952 & 56.593 \\
\hline
\end{tabular}

It is shown that the maximum and minimum deviation is $1.369^{\circ}, 0.952^{\circ}$ and $1.008^{\circ}, 0.996^{\circ}$ for wall angle of $76^{\circ}$ cone in experiment and simulation, respectively, and the maximum and minimum deviation is $4.509^{\circ}, 3.393^{\circ}$, and $2.806^{\circ}, 2.064^{\circ}$ for wall angle of $53.2^{\circ}$ cone in experiment and simulation, respectively.

Fig. (14) shows that the measurement of inclined wall for $76^{\circ}$ experimental cone, with $\alpha=77.314^{\circ}$ measured by LEXT OLS4100 Microscope. The angle measured by LEXT OLS4100 is $0.055^{\circ}$ less than by the Faro Gage.

\subsection{Thickness}

The experimental thickness measured using a micrometer and numerical thickness were compared to the analytical thickness calculated by Eq. (4) in (Fig. 15). 


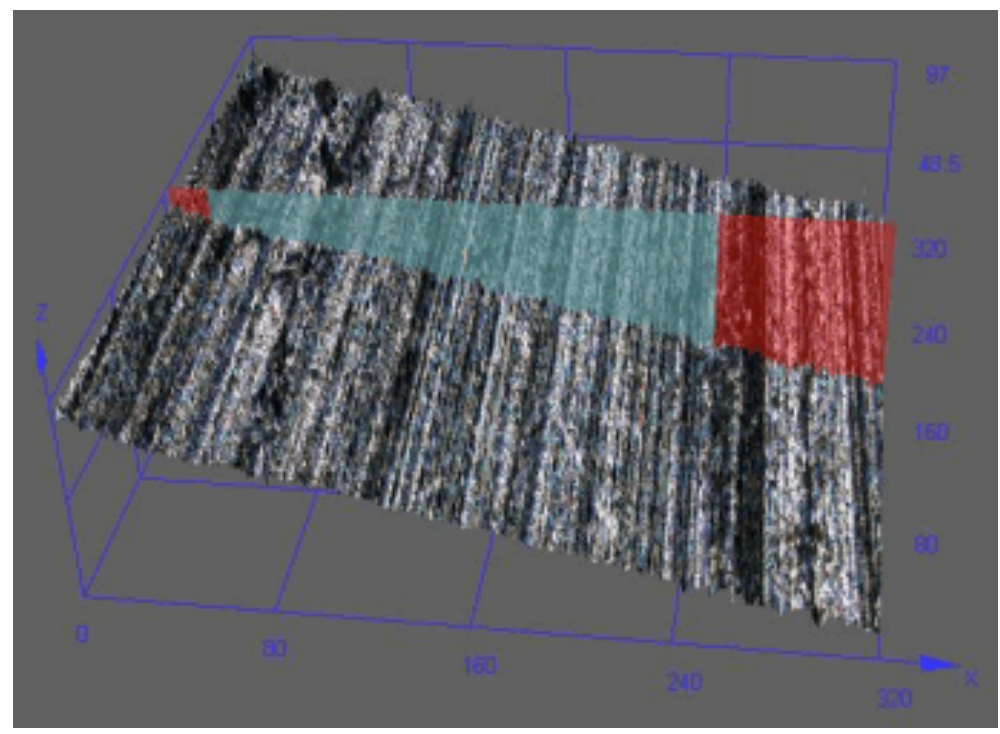

Fig. (14). The inclined wall of $76^{\circ}$ experimental cone.

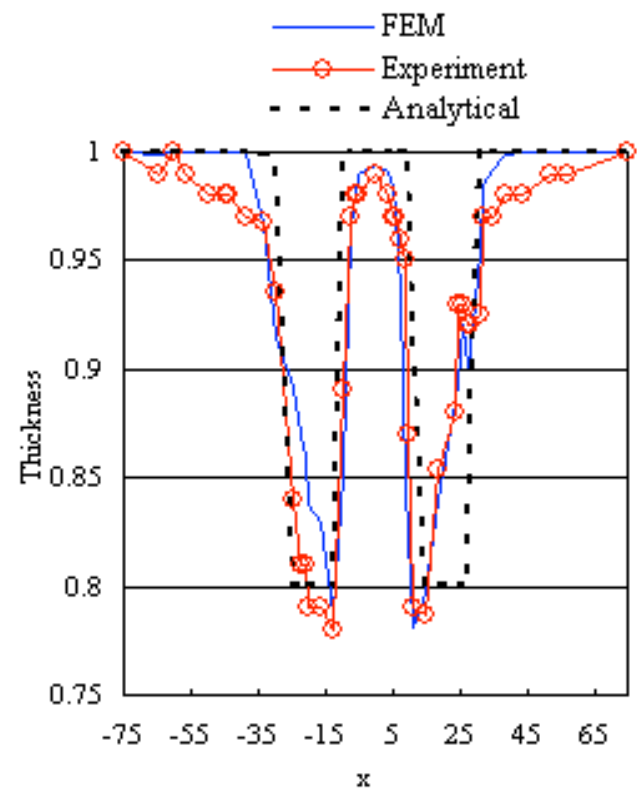

Fig. (15). Thickness comparison of simulation, experiment and theoretical calculation for $53.2^{\circ}$ cone.

Since, the initial thickness $t_{0}=1.0 \mathrm{~mm}$, it can be seen form Fig. 15 that the sheet thickness reduction occurred along the inclined wall. The thinnest thickness is $0.78 \mathrm{~mm}$ for $53.2^{\circ}$ cone in experiment. The thickness for base is $0.99 \mathrm{~mm}$.

\section{CONCLUSION}

A layered deformation analysis of DSIF is presented in this paper. The analysis includes the establishment of an equivalent layered model based on the contour trajectory and the calculation of the layered historical displacement under the assumption of straight projection. The relationship of displacement and contour forming layer is calculated by using of the translation of arc determined by the radius of tool. A general method which is proved to be the same as the sine law for cone is devised to estimate the thickness by using of the surface geometry of the desired component. Analytical, simulation and experimental results of the truncated cones with different height are compared for the historical displacement, the final geometry and thickness. From the comparative results, it is found that the analysis results coincide with the simulation results and experimental measurement. It is demonstrated the model and method proposed in this paper are efficient in the prediction of layered historical displacement, geometric accuracy and thickness for DSIF.

\section{CONFLICT OF INTEREST}

The authors confirm that this article content has no conflict of interest.

\section{ACKNOWLEDGEMENTS}

The authors would like to thank Zhejiang Provincial Natural Science Foundation of China under Grant No. LZ12E05003, the priority open fund from Zhejiang University of Technology under Grant No. 56310203039, the Science and Technology Department of Zhejiang Province under Grant No. 2010R50002-01 and No. 2012C01012-4 for their financial support.

\section{REFERENCES}

[1] R. Malhotra, J. Cao, M. Beltran, D. K. Xu, and J. Magargee, “Accumulative-DSIF strategy for enhancing process capabilities in incremental forming," CIRP Annals - Manufacturing Technology, vol. 61, pp. 251-254, 2012.

[2] J. M. Allwood, N. E. Houghton, and K. P. Jackson, "The design of an incremental sheet forming machine," Advanced Materials Research, vol. 6-8, pp. 471-478, 2005.

[3] B. Lu, J. Chen, H. Ou, and J. Cao, "Feature-based tool path generation approach for incremental sheet forming process," Journal of Materials Processing Technology, vol. 213, pp. 1221-1233, 2013.

[4] M. Rauch, J. Y. Hascoet, J. C. Hamann, and Y. Plenel, "Tool path programming optimization for incremental sheet forming applications," Computer-Aided Design, vol. 41, pp. 877-885, 2009.

[5] S. Tanaka, T. Nakamura, K. Hayakawa, H. Nakamura, and K. Motomura, "Residual stress in sheet metal parts made by incremental forming process,' In: Processdings Numiform 07, Portugal, 2007, pp. 475-480. 
[6] J. M. Allwood, D. Braun, and O. Music, "The effect of partially cut-out blanks on geometric accuracy in incremental sheet forming," Journal of Materials Processing Technology, vol. 210, pp. 1501-1510, 2010.

[7] M. Tisza, I. Panity, and P. Z. Kovacs, "Experimental and numerical study of a milling machine-based dieless incremental sheet forming," International Journal of Material, vol. 1, pp. 971-974, 2010.

[8] H. Meier, V. Smukala, O. Dewald, and J. Zhang, "Two point incremental forming with two moving forming tools," Key Engineering Materials, vol. 344, pp. 599-605, 2007.

[9] V. Franzen, L. Kwiatkowski, G. Sebastiani, R. Shankar, A. E. Tekkaya, and M. Kleiner, "Dyna-die: towards full kinematic in- cremental forming," International Journal of Material, vol. 1, pp. 1163-1166, 2008.

[10] Q. Wang, C. Yao, and W. Peng, "Research on experimental equipment for three point incremental forming," Light Industry Machinery, vol. 30, no. 5, pp. 13-15, 2012.

[11] C. F. Guzman, J. Gu, J. Duflou, H. Vanhove, P. Flores, and A. M. Habraken, "Study of the geometrical inaccuracy on a SPIF two slope pyramid by finite element simulations," International Journal of Solids and Structures, vol. 49, pp. 3594-3604, 2012.

[12] M. Bambach, "A geometrical model of the kinematics of incremental sheet forming for the prediction of membrane strains and sheet thickness," Journal of Materials Processing Technology, vol. 210, pp. 1562-1573, 2010.

(C) Wang et al.; Licensee Bentham Open.

This is an open access article licensed under the terms of the (https://creativecommons.org/licenses/by/4.0/legalcode), which permits unrestricted, non-commercial use, distribution and reproduction in any medium, provided the work is properly cited. 